\title{
High-resolution Electron Imaging and Spectroscopy of Reactive Materials and Liquid-Solid Interfaces in Energy Storage Devices
}

Michael J. Zachman ${ }^{1,2}$, Zhengyuan $\mathrm{Tu}^{3}$, Snehashis Choudhury ${ }^{4}$, Taylor Moon ${ }^{1}$, Qing Zhao ${ }^{4}$, Lynden A. $\operatorname{Archer}^{3,4}$, Lena F. Kourkoutis ${ }^{1,5^{*}}$

1. School of Applied and Engineering Physics, Cornell University, Ithaca, NY, USA.

2. Present address: Center for Nanophase Materials Sciences, ORNL, Oak Ridge, TN, USA.

3. Department of Materials Science and Engineering, Cornell University, Ithaca, NY, USA.

4. Robert Frederick Smith School of Chemical and Biomolecular Engineering, Cornell University, Ithaca, NY, USA.

5. Kavli Institute at Cornell for Nanoscale Science, Cornell University, Ithaca, NY, USA.

* Corresponding author: lena.f.kourkoutis@cornell.edu

Many modern energy devices rely on highly reactive materials, solid-liquid interfaces, or both, for their operation and performance. Physical and chemical processes at complex interfaces between dissimilar materials play a crucial role in catalysis, solar energy and fuel generation, and electrochemical energy storage, for example, but such interfaces are notoriously difficult to characterize at high spatial resolution in their native environment [1]. Typical approaches involve the removal of the liquid from such samples before characterization in high-vacuum instruments to prevent immediate evaporation. The associated washing and drying process can, however, result in structural and chemical modifications of soft or brittle materials present at the device interface [2]. Samples containing highly reactive materials, such as alkali metals, present additional challenges for characterization since exposed regions react quickly with air during transfer into a microscope or with contaminants in the vacuum during characterization, altering the sample's surface chemistry [3]. The lack of appropriate characterization methods for these materials in many cases limits our fundamental understanding of the performance of practical energy devices. Thus, development of techniques that give access to internal interfaces involving reactive materials and interfaces between liquids and solids, and provide structural, chemical and electronic information with nanometer resolution is key to progress in these areas.

Here, I will discuss our approach of cryogenic focused ion beam (cryo-FIB) milling and cryogenic scanning transmission electron microscopy (cryo-STEM) and spectroscopy to probe processes at interfaces in functional coin cell batteries. Cryogenic cooling of the samples offers multiple benefits in these systems. It allows liquids at solid-liquid interfaces to be preserved, chemically reactive samples to be transferred into the instrument without reaction with the environment, and clean FIB milling to be performed in materials where room temperature milling has proven problematic, such as in lithium. By coupling cryo-FIB milling of cross-sections with imaging and spectroscopy, e.g., SEM and EDX performed directly in the cryo-FIB, we demonstrate nanoscale structural and elemental characterization of complex interfaces in coin cell devices (Fig. 1). Importantly, once a cryo-immobilized device is loaded into the instrument, creating cross-sections by cryo-FIB milling is relatively straightforward which means that multiple areas on the device can be readily characterized and statistics of the observed features and interface processes can be build up. The cryo-FIB/SEM/EDX approach, therefore, allows us to bridge the significant size gap between the full device and the nanoscale features of interest.

Characterization of liquid-solid interfaces directly in the cryo-FIB is simple and fast but limited in resolution. For further improvements, we have developed cryo-FIB lift-out to prepare thin slices, or lamellas, from the frozen bulk sample. By combining cryo-FIB lift out with analytical cryo-STEM techniques we are able to provide high-resolution information about the interface structure in functional 
devices as well as track local changes in chemistry and bonding. This has allowed us to record the first nanoscale chemical maps of intact solid-liquid interfaces [4]. We have applied this technique to extract cross-sections of anode-electrolyte interfaces directly from functional lithium metal batteries, fully preserving all intrinsic structures, interphases, and liquids. Cryo-FIB in combination with cryoSTEM/EELS allows us to provide a direct picture of the nanoscale structure and composition of dendrites and their interphase layers (Fig. 2) [5]. Our results demonstrate that cryogenic electron microscopy provides new insights about processes inside energy devices by directly probing the structure and composition of unaltered reactive materials and intact interfaces at the nanoscale [6].

[1] F Zaera, Chem. Rev. 112 (2012), p. 2920.

[2] Y-Y Hu et al., Nat. Mater. 12 (2013), p. 1130.

[3] D-R Liu and DB Williams, Philos. Mag. B 53 (1986) p. L123.

[4] MJ Zachman, E Asenath-Smith, L Estroff, LF Kourkoutis, Microsc. Microanal. 22 (2016), p. 1338.

[5] MJ Zachman, Z Tu, S Choudhury, LA Archer, LF Kourkoutis, Nature 560 (2018), p. 345.

[6] Supported by NSF (DMR-1654596, DMR-1429155, DMR-1719875) and the Packard Foundation.
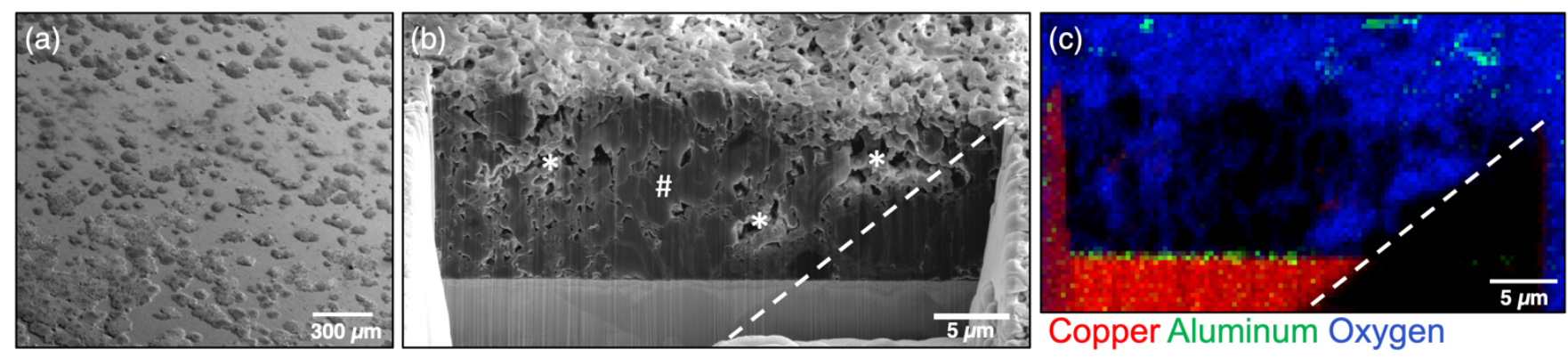

Figure 1. Chemical mapping of interfaces in reactive materials by cryo-FIB/EDX. (a) Cryo-SEM image of lithium deposited on a copper coin cell electrode. The presence of a $\sim 15 \mathrm{~nm}$ thick alumina layer on the electrode results in localized lithium deposits. (b) Cross-sectional cryo-FIB milling allows internal dense (\#) and porous $(*)$ regions to be identified. (c) Chemical mapping of the interface is enabled by cryo-EDX on these cross-sections. Shadowing due to detector placement is indicated by the dashed lines.
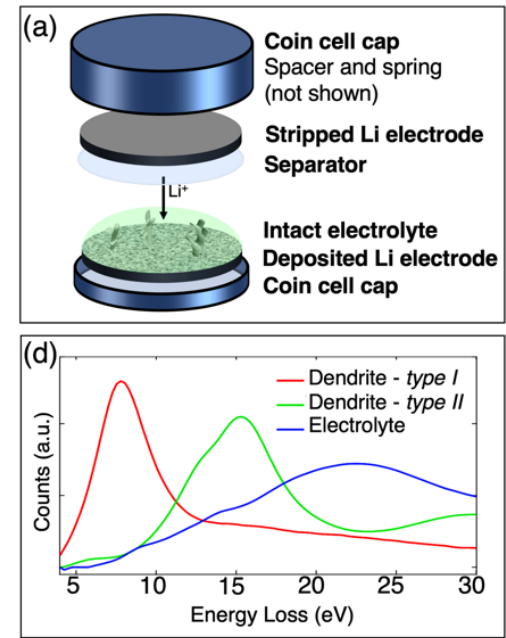
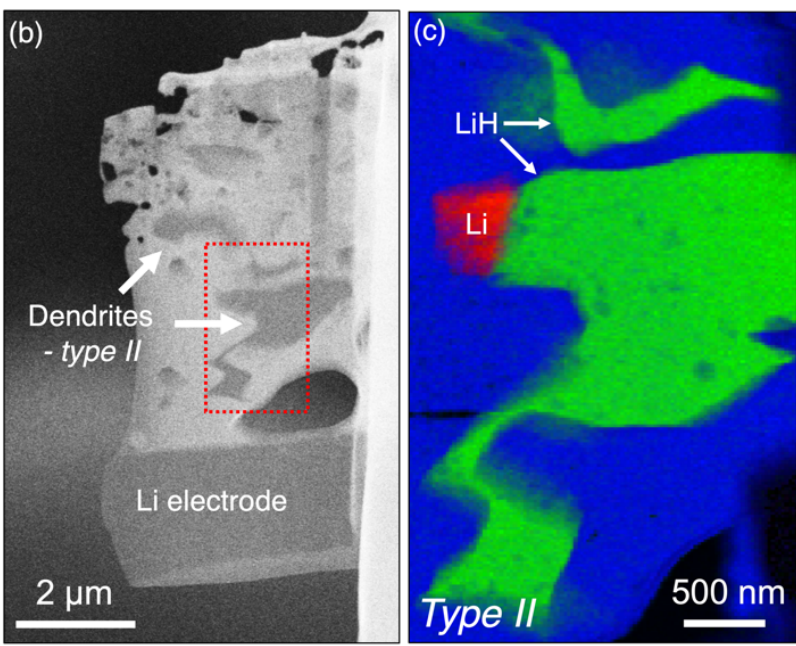

Figure 2. Nanoscale mapping of dendrites in lithium metal coin cell battery (a) enabled by cryo-FIB lift-out and cryo-STEM. (b) CryoSEM image of an electron transparent lamella captures the electrolyte-electrode interfaces and slices through dendrites which formed during the charge-discharge cycling of the device. (c-d) Cryo-STEM/EELS mapping reveals the composition and bonding of two types of dendrites, one of which unexpectedly consists of lithium hydride and may contribute disproportionately to capacity fade [5]. 